\title{
Path Quality Estimator for 802.15.4e TSCH Fast Deployment Tool
}

\author{
José Vera-Pérez, David Todolí-Ferrandis, Salvador Santonja-Climent, \\ Javier Silvestre-Blanes, and Víctor Sempere-Paya
}

\begin{abstract}
- this paper introduces a novel quality estimator that uses different metrics to decide the best path towards the root in Wireless Sensor Networks. The different metrics are assessed at medium access control layer (MAC), under the IEEE 802.15.4 standard, and are used at network layer, enhancing the best path selection process done by the routing protocol, and at the application layer, enabling visual quality indicators in the nodes. This quality function is used during deployment stage; ensuring nodes are located optimally and nimbly. This mechanism will help WSN's adoption in Industrial Internet of Things applications.
\end{abstract} WSN.

Keywords - Deployment, link quality, objective function,

\section{INTRODUCTION}

$\mathrm{O}$ $\mathrm{NE}$ of the enabling technologies of the emerging Industrial Internet of Things (IIoT) are the Wireless sensor networks (WSN): systems formed by a multitude of sensors distributed in a geographical extension (to a greater or lesser scale), that perform a distributed collection of information (environmental, physical, gases, biological...) and communicate it to a central system using wireless technologies. This creates high granularity sensory systems with a much lower cost than the wired solutions. The possibilities offered by gathering large amounts of data, in real time and at a minimal cost, open the door to a wide range of applications for the WSN regarding not only industry, but also environment, public safety, smart cities, infrastructure, and many more. Traditionally, for a WSN to operate reliably in harsh environments, pre-deployment studies/tools and additional management devices are necessary. Nevertheless, an easy, rapid and more efficient network deployment avoiding this

Paper received March 20, 2018; revised April 23, 2018; accepted April 25, 2018. Date of publication July 31, 2018. The associate editor coordinating the review of this manuscript and approving it for publication was Prof. Grozdan Petrović.

This paper is a revised and expanded version of the paper presented at the 25th Telecommunications Forum TELFOR 2017 [16].

This work is supported by IVACE (Insituto Valenciano de Competitividad Empresarial) through FEDER funding (exp. IMDEEA/2017/103).

D. Todolí-Ferrandis, S. Santonja-Climent and J. Vera-Pérez are with the Instituto Tecnológico de Informática (ITI), Camino de Vera $\mathrm{s} / \mathrm{n}$, 46022 Valencia, Spain (e-mail:[dtodoli,ssantonja,jvera]@iti.es).

J. Silvestre-Blanes and V. Sempere-Payá are with the Universitat Politècnica de València (UPV), Camino de Vera s/n, 46022 Valencia, Spain (e-mail: jsilves@disca.upv.es, vsempere@dcom.upv.es). pre-deployment phase is bound to overcome the arguments wielded by technology hesitant adopters. A solution proposed is based in adding mechanisms and tools in each node that enable triggering a connectivity and quality test, and showing the results in a user-friendly manner during the deployment.

The objective of this paper is to define a quality estimator that allows characterizing the links and paths between nodes during the first steps of deployment. This allows the Routing Protocol for Lossy networks (RPL) to create rapidly a robust topology using the quality estimator as metric input for the objective function that resolves the best paths for each node in the network. The quality assessment is designed to consider and merge different indicators, such as signal strength (RSSI), expected transmission count (ETX) and hop count, and mapped together using fuzzy logic and statistics, making the impact of each variable tunable to enhance easier optimization for a wide range of applications. In addition, the resolution algorithm is embedded in the RPL mechanism, avoiding any extra control packets overhead and optimizing the software implementation. This quality parameter also supports the operator during deployment tasks, by enabling the node to represent the value of connection quality and allowing finding optimal locations to place sensor nodes. Therefore, it will reduce the time taken to deploy the network, as well as simplifying operator's work, meaning staff without technical knowledge is able to deploy a WSN without simulators, planning software or any other external complex tools.

The rest of the paper is organized as follows: Section II shows related work concerning multipurpose link quality estimation used for the RPL objective functions. In Section III, we define the chosen parameters for the link quality estimator, and their mapping for the RPL objective function. In Section IV, the different simulations and results are summarized, comparing the response of the network to different quality calculation configurations. Finally, we conclude this paper in Section V.

\section{RELATED WORK}

The Internet Engineering Task Force (IETF) ROLL(Routing Over Low power and Lossy networks) has overseen developing and standardizing the RPL protocol and has so far proven to be a powerful and flexible route selection mechanism for Low Power and Lossy Networks (LLNs). Therefore, both the scientific and industrial community has focused on it and many studies have been published evaluating their performance [1]. 
The protocol uses different metrics introduced in the Objective Function (OF) to characterize each of the links and to select the best of the present routes. The selection of an optimum metric for resolving routes through the $\mathrm{OF}$ is still a reason for study and that is why different implementations have been developed evaluating the characteristics of different metrics.

The IETF ROLL group has also been responsible for developing two OFs that are used by default in the RPL protocol, the Objective Function Zero (OF0) [2] and the Minimum Rank with Hysteresis Objective Function (MRHOF) [3]. There are different studies and analyses that compare the performance of both OFs. In [4] the performance of these two OFs is evaluated considering several parameters for the two OFs defined in RPL, such as network convergence time, power consumption, ETX, number of jumps, PDR and latency. They conclude that MRHOF has a better performance than OF0 in terms of network quality, with MRHOF being more suitable for applications where data delivery and network reliability are the priority, while OF0 is more suitable for applications that require rapid network formation and lower power consumption. Kamgueu et al [5] compared ETX with a solution based on the remaining energy of the nodes that form the network, proving that it manages to extend the useful life of the network. Kim et al [6] deal with load balancing and congestion problems using RPL information on packet queues of different nodes. In [7] Gonizzi et al propose a metric that minimizes the delay, comparing its performance again with ETX. In [8] the IETF group defines some of the metrics used for the calculation of routes that can be used in RPL and [9] presents a survey that details different metrics that can be used to evaluate link quality, differentiating between hardware-based (RSSI, LQI, SNR) and software-based metrics (PRR and ETX). This survey concludes that the use of a single metric can only evaluate a single property of the link, and provides functions that combine some of these metrics.

Karkazis et al [10] propose some methods to quantify different routing metrics and different ways of combining them. Baccour et al [11] propose F-LQE, a link quality estimation based on four properties: packet delivery, asymmetry, stability and channel quality. The quality of the link is specified based on fuzzy rules. The results demonstrate a higher performance compared to other existing solutions. In [12] Rekik et al point out that ETX is not accurate enough and they propose an alternative metric based on holistic estimation of link quality, where several link metrics are combined. Gaddour et al [13] propose OF-FL, an OF that combines a set of metrics to provide a configurable routing decision.

The proposed solution for estimating communication quality during WSN deployment introduced in this article uses some of the aforementioned metrics, but includes new sampling policies for some metrics to better adapt to the MAC layer selected, the IEEE802.15e standard operating in Time-Slotted Channel Hopping (TSCH) mode, while avoiding the introduction of any overhead due to extra control traffic.

\section{Proposed SOLUTION}

The solution proposed in this paper uses the link quality estimator for different purposes. These values obtained from the quality function can be used as an indicator in those situations to find the best location for the devices during the deployment phases of a wireless sensor network (WSN). On the other hand, the quality metric is used in the RPL OF to improve the decision-making of the routing protocol, ensuring that the quality informed to the user matches the underlying network topology.

Devices that have the ability to establish wireless communications links should be able to assess the quality of the path through which the information is routed, in order to establish robust and highly reliable communications, characteristics necessary in the aggressive conditions found in industrial environments. As a mechanism for evaluating the quality of communications, the nodes will have the ability to estimate the level of link quality with surrounding neighbors, allowing the configurations and topologies that are formed in the network to improve the reliability and robustness of communications. There are important aspects to optimize, such as network coverage, message latency or topology stability, which are important to maintain a certain link quality and the service provided by the network. In practice, these metrics usually come into conflict, forcing to reach a compromise when optimizing the general operation of the sensor network.

This quality evaluation mechanism may be used as follows:

First, allow the operator to know the most optimal location for placing the nodes, representing in a simple way different quality values depending on the data that the node picks up during the deployment phase. Secondly, quality levels are used as metrics to perform routing tasks, in order to choose the paths that present a higher level of quality and thus improve efficiency when retransmitting messages through the mesh network.

The propagation of radio signals can be affected by several factors that contribute to the degradation of their quality. Some of these metrics will depend only on the radio environment and the hardware configuration available to the devices, and in other cases, it will depend on the topology of the network and on the planning of radio resources.

Among all these parameters, some have been chosen that will be combined to obtain a multi-objective quality estimator, which will allow evaluating different aspects of the quality of the links:

The RSSI parameter, which represents the signal level with which they are receiving the packages from the different neighbors within the range of coverage, being a faithful indicator of the quality of the RF communications links.

The parameter ETX, which represents the number of retransmissions necessary for a packet to be transmitted correctly;

Finally, the number of jumps from one node to the root has been used, which can be a proportional and approximate parameter of the latency between a node and the root, in a deterministic and synchronized network, without any additional traffic. 
Using fuzzy logic operations, these metrics have been sampled using a series of mapping functions, with the aim of normalizing the different metrics within a same range delimited between 128 and 512. For each of the metrics used, filtering is performed. Exponential Weighted Moving Average (EWMA) type to smooth changes and improve stability, but ensuring that the averaged variance is close to the most recent data. Once the samples for each of the metrics are obtained, they are combined using a weighted arithmetic average with different weights for each metric, which will allow to easily modify the impact of each of the metrics on the result of the quality estimate.

The mapping range for the different metrics has been chosen to follow the same philosophy that is used in current implementations of the ETX metric and the RPL MRHOF objective function. These limits prevent the use of floating point format values, which can overload memory and CPU utilization in this type of restricted devices typical of WSN. The objective function MRHOF normalizes the ETX metric by multiplying it by a factor of 128 and since the minimum value of this metric is 1 , the ranges of values used by this function will move between 128 and infinity. As an upper limit, different studies recommend not using links that have an ETX equal to or greater than 4, corresponding to a normalized value of 512 , since it would imply $75 \%$ of packets are being lost. The process of estimating each metric is detailed below.

\section{A. Received Signal Strength Indicator}

The RSSI metric represents the signal level strength of a received packet. It is a parameter directly measured by the hardware of the device whenever a message is received. There are other parameters such as the LQI or the SNR that represent the quality of the channel, however RSSI is chosen because it is faster to get a reliable channel representation, whereas the LQI has a greater variance and the SNR requires more stages and computational resources when calculating the noise of the channel [9].

In the networks of wireless sensors deployed, under the IEEE 802.15.4 standard, and using RPL as a dynamic routing protocol, several types of messages are exchanged: beacons and Keep-Alives, ICMPv6 DIO messages (DODAG ${ }^{1}$ Information Object), DIS messages (DODAG Information Solicitation) and DAO messages (Destination Advertisement Object), or data messages using UDP, as well as all acknowledgment messages (ACK). All these types of messages mentioned are used to obtain the level of RSSI with which these messages arrive.

Unlike other solutions that use RSSI values to estimate channel quality, this solution allows nodes to measure the RSSI value for each of the channels involved in the communication. This is relevant because the devices have been developed under the IEEE 802.15.4e standard using the TSCH mode that is specially designed to work in industrial environments. This mode allows the communication to alternate between different frequency channels to mitigate negative effects of the channel, such as interference or fading. As a result, the level of RSSI will vary over time, because not only a given channel changes over time, but the communication also jumps

${ }^{1}$ Destination-Oriented DAG (Directed Acyclic Graph) between channels over time. Therefore, when evaluating the RSSI level of a received message, the channel through which the message has been transmitted is also verified, allowing the characterization of this metric to reflect this time/frequency variability. In order to organize all this information, in each of the nodes a series of matrixes have been defined to store the RSSI measured values for each of the neighbors that are within the range, and for each of the channels used in TSCH. Fig. 1 shows a representation of the process of updating values in the commented matrixes. Each RSSI value for each channel (matrix cells) is computed and statistically softened with the EWMA filter, which allows considering past values, to improve stability, and then the corresponding cell of the matrix is updated. This type of filtering uses a structure like the one shown in (1).

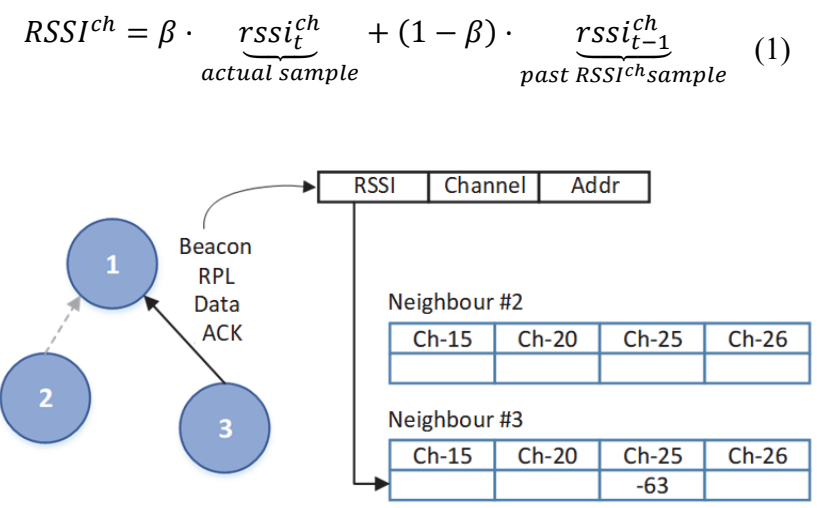

Fig. 1. RSSI metric value update diagram.

TABLE 1: RSSI COMPUTING MATRIX FOR EACH NEIGHBOR IN A NODE.

\begin{tabular}{|c|c|c|c|c|c|}
\hline & $R S S I^{c h \_1}$ & $\ldots$ & $R S S I^{\text {ch_n }}$ & \multirow{5}{*}{$\Rightarrow$} & $\begin{array}{c}\text { RSSI }(n=\text { number } \\
\text { of channels })\end{array}$ \\
\hline \multirow{2}{*}{ Node A } & \multirow{2}{*}{$R S S I_{\text {nodeA }}^{c h \_1}$} & \multirow{2}{*}{$\ldots$} & \multirow{2}{*}{$R S S I_{\text {nodeA }}^{c h \_n}$} & & $\sum_{c h=1}^{n} R S S I_{\text {node } A}$ \\
\hline & & & & & $n$ \\
\hline \multirow[t]{2}{*}{ Node B } & \multirow[t]{2}{*}{$R S S I_{\text {nodeB }}^{c h \_1}$} & \multirow{2}{*}{$\ldots$} & \multirow{2}{*}{$R S S I_{n o d e B}^{c h \_n}$} & & $\sum_{c h=1}^{n} R S S I_{\text {node } B}$ \\
\hline & & & & & $n$ \\
\hline
\end{tabular}

Then, for each row (representing a link with a neighbor in all available channels), the mean is applied to obtain a meaningful but single value to feed the quality estimator (Table 1). In this case, $\beta$ is set between 0.3 and 0.15 depending on the freshness of the actual sample (time limit set to 10 minutes). This allows to characterize each of the channels involved in the communication, allowing, for example, to perform a blacklisting of those channels that are hindering communication. The mapping function to introduce RSSI in the quality function is shown in Fig. 2. Map function for RSSI metric

\section{B. Expected transmission count}

ETX is a link quality measure between two nodes in a wireless network based on the exchange of data packets. This metric is commonly used in routing algorithms in mesh networks, for example in the MRHOF RPL objective function, to minimize packet loss. The ETX is defined mathematically as shown below (2), where $\mathrm{N}$ means the number of packets sent or received, and subscripts refer to node $i$ as the sender, and $j$ as the receiver: 


$$
E T X_{i j}=\frac{N_{i}}{N_{j}}
$$

In this case, ETX values are not resolved for each channel due to the very nature of the ETX metric. ETX counts the number of retransmissions needed to transmit correctly a packet over a link, but in TSCH, retransmissions are made in different channels due to the channel hopping, so it is not possible to give an accurate value of ETX for a particular channel. ETX calculated values are also filtered with EWMA. The mapping feature for the ETX metric, used in the quality function is shown in Fig. 3. As an alternative, PER (Packet error rate) could be used to measure how many packets are lost in each of the channels, however, this would cause the time needed to process this metric to be proportionally larger, depending on the percentage of channels used, compared to the method of obtaining the ETX metric.

\section{Number of hops}

The metric number of hops in the quality function aims to provide an approximate and simplified value of the point-to-point delay between two nodes. An exact implementation of this parameter would require an additional exchange of information, in addition to keeping track of the packets all the way through the WSN. This would cause heavy network congestion and have a negative impact on the complexity of the implementation.

Although it is a simple assumption, it can be justified since the MAC layer follows a synchronized and deterministic behavior governed by $\mathrm{TSCH}$, with a data packet generation rate (6 seconds/packet) much higher than the duration of slot frames scheduled at MAC layer. Each generated packet needs one slot per hop to be transmitted. Since we use frames composed of 7 slots of 10 milliseconds, 85 slot frames fit within one period, leaving a sufficient margin to forward any packets remaining in the node's queue. Therefore, a packet end-to end delay can be assumed to correspond with one frame duration per hop up to the root. It is true that this assumption fails to consider retransmissions of failed packets, but in the overall quality estimation, this is palliated by the impact of ETX. The function of mapping of the number of hops used in the quality function is shown in Fig. 4.

To obtain the number of hops, it is necessary to use a variable that is incremented with each hop and retransmitted to the leaf nodes so that they know their situation within the RPL topology. However, we want to avoid transmitting additional control traffic that could overload the network.

Therefore, we use the RPL control messages to include information about this metric, in order to distribute it through the topology. The RPL control messages (DIO, DAO, DIS) are used to build and maintain an RPL topology, with the aim of transmitting the necessary information so that the nodes can choose the routes through which to route the information. In this way, the range is propagated and updated in one of the fields of these control messages. The different RPL control messages also allow to include information about different metrics in headers that are embedded in messages, called
Metric Containers. In this way, we can include a variable that is increasing a fixed value with each jump, propagating these messages down the tree topology, allowing each node to calculate the distance in hops to the root.

Fig. 5 depicts a representation of the message exchange for the calculation of the number of jumps. These discrete values are not affected over time, with the exception of changes in the topology; therefore, it will not be necessary to perform smoothing as we do with the other two metrics.

\section{Quality estimator}

Equation (3) displays the calculation of the quality estimation as a combination of the weighted values of the three metrics used. $L Q S$ variable represents link quality, $\mu_{i}$ represent the mapped values of the different metrics and $\alpha_{i}$ represent the weights assigned to each of the metrics.

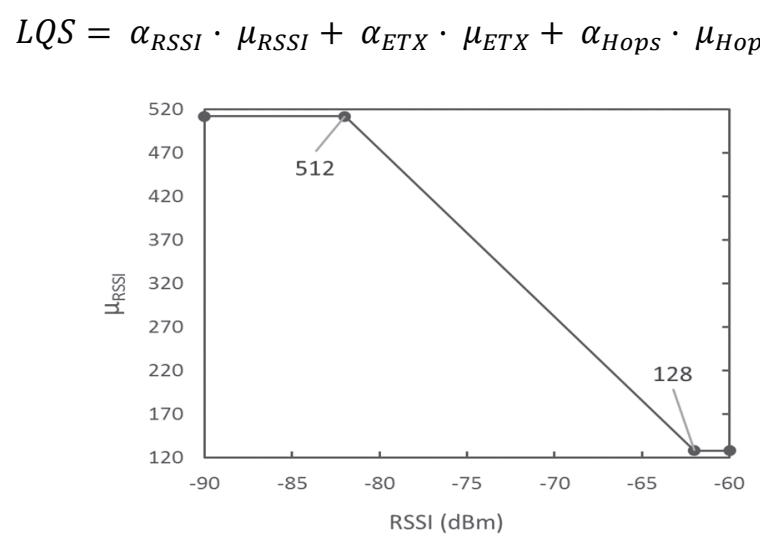

Fig. 2. Map function for RSSI metric.

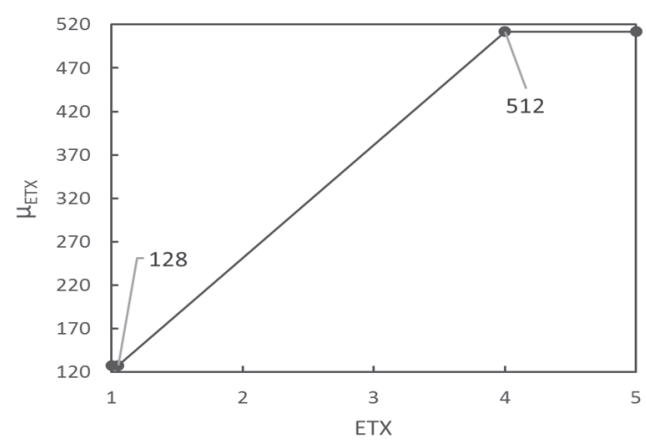

Fig. 3. Map function for ETX metric.

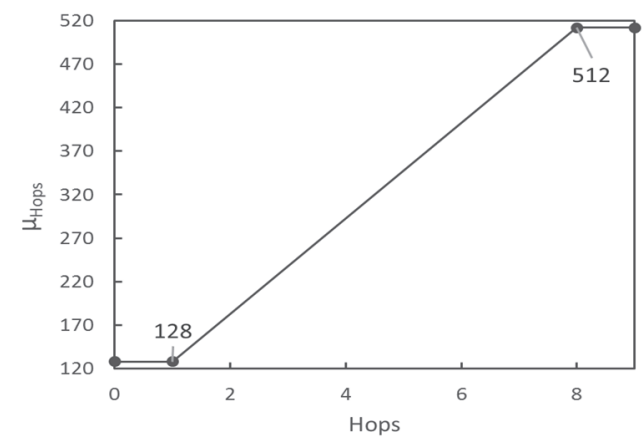

Fig. 4. Map function for Hop metric.

\section{E. Hysteresis Mechanism}

Because the quality estimate will be used to make decisions when choosing the best routes, it is necessary to implement a hysteresis mechanism, just as it is done in the MRHOF objective function [3]. This mechanism allows 
improved stability in parent changes in situations where the metrics of several neighbors have a similar range.

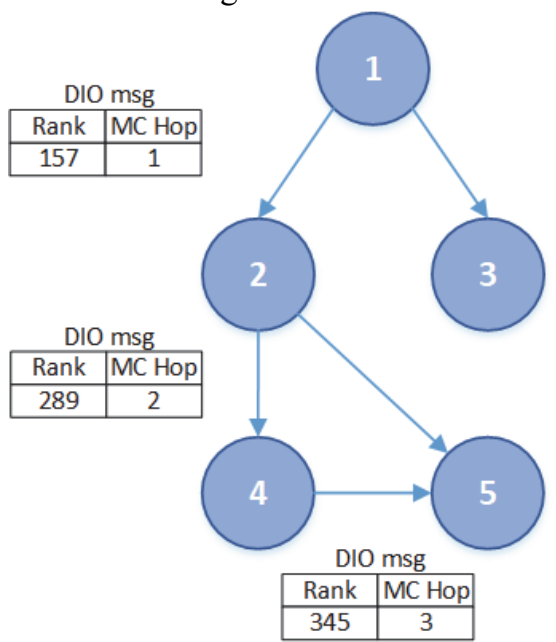

Fig. 5. DIO message exchange mechanism.

In this implementation, having a quality estimator using different metrics, using a single threshold does not reliably represent a value that is consistent with the different metrics. In order to implement this, we use the same weights that have been used in (3), with thresholds applied to each metric individually. The margins that have been chosen will allow acting against changes with a certain level of precision. In ETX a margin of 0.75 has been chosen, and $2 \mathrm{~dB}$ for the RSSI metric. For the number of hops, given its nature, threshold is 1 hops, which is the same as no hysteresis.

\section{Simulation AND Results}

In order to perform tests and experiments, the Cooja simulator integrated in Contiki has been used. The nodes selected are Zolertia Z1, whose msp430 microcontroller can be emulated in Cooja by the underlying tool MSPSIM, with some modifications to enable TSCH with the initially incompatible $\mathrm{CC} 2420$ transceiver. The nodes are configured with a TSCH schedule based on a modification of Minimal 6TiSCH [14], using slot frames of 7 slots size with 3 active slots.

The topology chosen sets forces a minimum of 3 hops in the farthest nodes, preventing a star configuration and ensuring a multi-hop network. This scenario can be seen in Fig. 6 , with a $100 \times 100 \mathrm{~m}$ grid, each node has a $50 \mathrm{~m}$ RF range, and the separation between nodes in range is of $30 \mathrm{~m}$ or $42^{\prime} 2 \mathrm{~m}$. Normally, a node connects directly with a parent of its top level, but there are cases in which the number of hops to the sink exceeds three if the accumulated metric of the top-level nodes within range is worse than the metric of neighboring nodes of the same level. To compare the packet loss, and delay of the network and test the implemented RPL's quality estimator objective function represented in Equation (3), different weight ( $\left.\alpha_{\mathrm{ETX}}, \alpha_{\mathrm{RSSI}}, \alpha_{\mathrm{Hops}}\right)$ configurations have been set up, and 20 simulation iterations for each configuration have been run.

The following Fig. 7 and Fig. 8 show the results obtained for 5 different configurations: MRHOF is used for comparison as is the preconfigured one in Contiki.

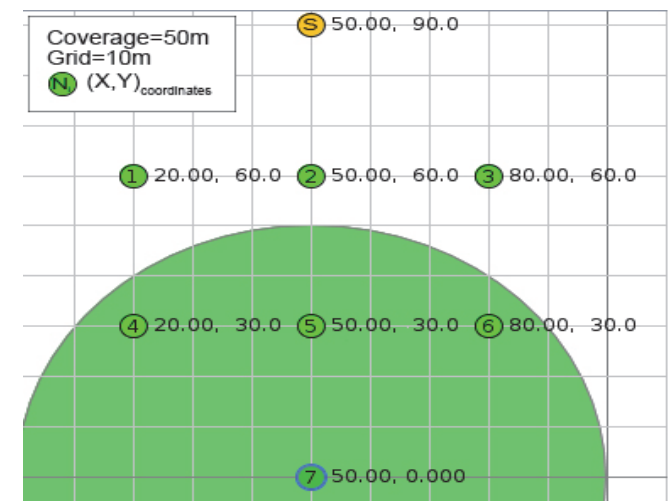

Fig. 6. Simulated network.

Then the proposed LQS OF is set with $\alpha_{i}$ in order to check the impact of each parameter; and giving equal weight to each parameter in order to test the improvements of combined metrics.

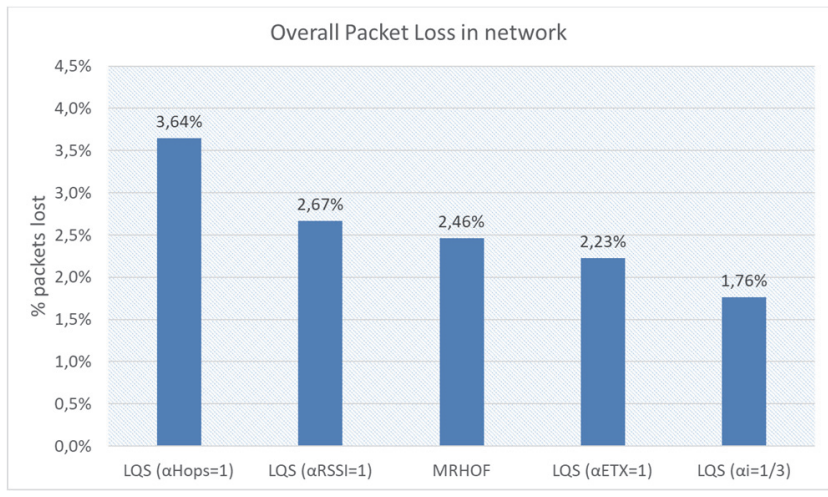

Fig. 7. Packet loss for the 5 OF configuration simulated.

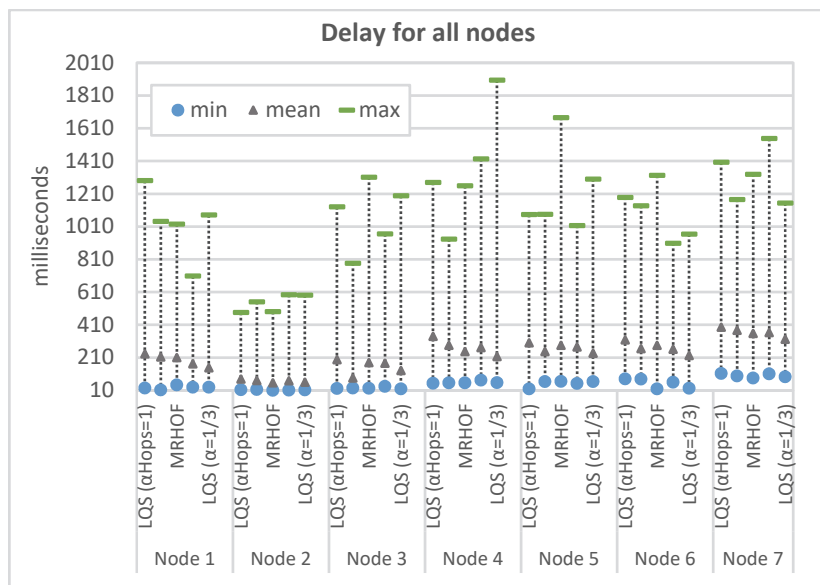

Fig. 8. Delay performance in every node for the $5 \mathrm{OF}$ configuration simulated.

Smaller losses are obtained in the LQS configuration $\left(\alpha_{E T X}=1\right)$ if we compare it with the MRHOF function, whose behaviour should be similar, since both use only the ETX metric. The only difference between these two configurations (MRHOF and LQS $\left(\alpha_{E T X}\right)$ ) is the maximum limit allowed for the link metric, configured as 1024 in the implementation of MRHOF, and 512 for LQS, as indicated in RFC6719. This causes the same metric to behave slightly differently since MRHOF presents some more losses because it allows communication through links with lower quality. 
The results show that the configuration that combines the three metrics $\left(\alpha_{i}=1 / 3\right)$ presents a reduction in the percentage of packet loss, while maintaining a lower average delay than for the rest of the configurations (Fig. 8 shows the statistics of delay for the 7 nodes of the network in the 5 simulated configurations). The values of packet loss that have been obtained are quite low in all cases, due to the deterministic medium access (TSCH), but still getting improvements close to $1 \%$ in that interval with respect to the rest of configurations.

Regarding the average delay, if we focus only on the node 7 , which is the spatially furthest from the coordinator and therefore with the most critical values, the configuration that uses a combination of the three metrics presents a result approximately 3 milliseconds less than the rest of configurations. In addition, analyzing the results obtained for LQS $\left(\alpha_{\text {HOPS }}=1\right)$, which should be the ones with the lowest delay value, it has been concluded that this metric should be replaced by a better approximation representing the delay point a point, which will be reflected in a shorter delay for the LQS objective function that balances the three metrics.

Finally, in this last graph of Fig. 9 the parent changes that occur during the simulations of the different configurations are shown to adapt the topology to changes in quality. As can be seen in the figures, the configuration that uses a combination of the three metrics (LQS $\left(\alpha_{i}=\right.$ $1 / 3)$ ) is more sensitive to the quality changes of the links, allowing to adapt better to choose the paths that present a more optimal level. In the case of the configuration that uses only the number of hops, we see that there are no changes of parent. This is because the metric of the number of hops is not a variable that changes with the quality of the link, but it will always choose the shortest paths, except for the addition of new nodes that allow reaching the coordinator in fewer jumps.

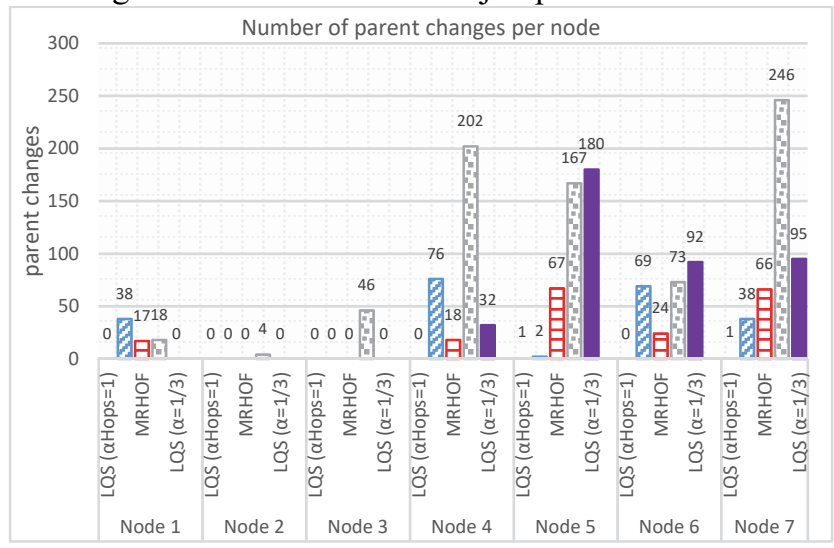

Fig. 9. Number of parent changes per node for the $5 \mathrm{OF}$ configuration simulated.

\section{CONCLUSION}

This paper presented a quality estimator and its application as routing metric for RPL networks to support fast deployments. The objective of the proposed metric is to ensure reliable end-to-end delivery in industrial environments through efficient link quality estimation. The proposed metric combines three properties, namely packet retransmissions, hops and channel RSSI, without requiring extra control traffic. The metric was evaluated through COOJA simulations, demonstrating its outperformance over using single metrics, and the impact each parameter can have in the overall path's quality, in terms of packet loss ratio, end-to-end delay. Further experiments will comprise the addition of energy metrics for longer network lifetimes in steady operation, a better metric to characterize end-to-end delay, and changing OFs during runtime, as described in [15].

\section{REFERENCES}

[1] O. Gaddour, A. Koubâa, S. Chaudhry, M. Tezeghdanti, R. Chaari and M. Abid, "Simulation and Performance Evaluation of DAG Construction with RPL," in IEEE Third International Conference on Communications and Networking (ComNet), pp. 1-8, 2012.

[2] IETF, "RFC 6552 - Objective Function Zero for the Routing Protocol for Low-Power and Lossy Networks (RPL)," 2012.

[3] IETF, "RFC 6719 - The Minimum Rank with Hysteresis Objective Function," 2012.

[4] N. Pradeska, Widyawan, W. Najib and S. S. Kusumawardani, "Performance Analysis of Objective Function MRHOF and OF0 in Routing Protocol RPL IPv6 Over Low Power Wireless Personal Area Networks (6LoWPAN)," in 8th International Conference on Information Technology and Electrical Engineering (ICITEE), Yogyakarta, Indonesia, 2016

[5] P. O. Kamgueu, E. Nataf, T. D. Ndié and O. Festor, "Energy-based routing metric for RPL," Doctoral dissertation, INRIA, 2013.

[6] H.-S. Kim, J. Paek and S. Bahk, "QU-RPL: Queue utilization based RPL for load balancing in large scale industrial applications," in 12th Annual IEEE International Conference on Sensing, Communication and Networking (SECON), Seattle, WA, USA, 2015.

[7] P. Gonizzi, R. Monica and G. Ferrari, "Design and evaluation of a delay-efficient RPL routing metric," in 9th International Wireless Communication and Mobile Computing Conference (IWCMC), Sardinia, Italy, 2013

[8] IETF, "RFC 6551 - Routing Metrics Used for Path Calculation in Low-Power and Lossy Networks," 2012.

[9] N. Baccour, A. Koubâa, L. Mottola, M. A. Zúñiga, H. Youssef, C. A. Boano and M. Alves, "Radio link quality estimation in wireless sensor networks: A survey," ACM Transactions on Sensor Networks (TOSN), vol. 8 (4), 2012

[10] P. Karkazis, H. C. Leligou, L. Sarakis, T. Zahariadis, P. Trakadas, T. H. Velivassaki and C. Capsalis, "Design of primary and composite routing metrics for RPL-compliant Wireless Sensor Networks," in International Conference on Telecommunications and Multimedia (TEMU), Chania, Greece, 2012.

[11] N. Baccour, A. Koubâa, H. Youssef, M. B. Jamâa, D. d. Rosário, M. Alves and L. B. Becker, "F-LQE: A Fuzzy Link Quality Estimator for Wireless Sensor Networks," in European Conference on Wireless Sensor Networks (EWSN), Coimbra, Portugal, 2010.

[12] S. Rekik, N. Baccour, M. Jmaiel and K. Drira, "Holistic link quality estimation-based routing metric for RPL networks in smart grids," in IEEE 27th Annual International Symposium on Personal, Indoor, and Mobile Radio Communications (PIMRC), Valencia, Spain, 2016.

[13] O. Gaddour, A. Koubaa, N. Baccour and M. Abid, "OF-FL: QoSaware fuzzy logic objective function for the RPL routing protocol," in 12th International Symposium on Modeling and Optimization in Mobile, Ad Hoc, and Wireless Networks (WiOpt), Hammamet, Tunisia, 2014.

[14] IETF, "RFC 8180 - Minimal IPv6 over TSCH Mode of IEEE 802.15.4e (6TiSCH) Configuration," 2017.

[15] M. G. Amor, A. Koubâa, E. Tovar and M. Khalgui, "Cyber-OF: An Adaptative Cyber-Physical Objective Function for Smart Cities Applications," in 28th Euromicro Conference on Real-Time Systems (ECRTS), Toulouse, France, 2016.

[16] J. Vera-Pérez, D. Todolí-Ferrandis, J. Silvestre-Blanes, S. SantonjaCliment and V. Sempere-Paya, "Path quality estimator for wireless sensor networks fast deployment tool," 2017 25th Telecommunication Forum (TELFOR), Belgrade, 2017, pp. 1-4. 